\title{
Reducing Cross-Coupling in a Compliant XY Nanopositioner for Fast and Accurate Raster Scanning
}

\author{
Yuen Kuan Yong, Kexiu Liu, and S. O. Reza Moheimani, Senior Member, IEEE
}

\begin{abstract}
A compliant $X Y$ nanopositioner is presented in this brief. The device is designed to have a very low cross-coupling between the $X$ - and $Y$-axis. Despite this, during high-speed raster scans, the cross-coupling effect can not be ignored. In this brief, a $\mathrm{H}_{\infty}$ controller is designed and implemented to minimize the $X$-to- $Y$ cross-coupling of the nanoscale positioning stage, particularly at its mechanical resonance frequencies. The controller is augmented with integral action to achieve accurate tracking, as well as sufficient damping. Raster scan results over an area of $10 \mu \mathrm{m} \times 10 \mu \mathrm{m}$ with small positioning errors are demonstrated. High-speed accurate raster scans of up to $100 \mathrm{~Hz}$, with nanoscale resolution are also illustrated.
\end{abstract}

Index Terms-Compliant $X Y$ nanopositioner, cross-coupling, H-infinity control, high-speed scans, raster scanning.

\section{INTRODUCTION}

$\mathbf{T}$ HE RAPIDLY growing and ever-increasing applications of nanotechnology have increased the demand for high-speed and high-precision nanopositioning systems. The emergence of compliant, piezoelectric stack-actuated nanopositioners fulfils the requirements of nanotechnology related applications. These applications include scanning probe microscopy (SPM) [1], nano-metrology [2], tracking and analysis of biological cell events [3], nano-indentation for high-density data storage systems [4]-[6], and beam steering for optical communication systems [7].

Cross-coupling effect is one of the main complications associated with scanning applications in atomic force microscopy (AFM). AFMs utilize sharp probes of few atoms wide (located at the end of a flexible micro-cantilever) and nanopositioning scanners that move samples relative to the probe to perform raster scans over the sample surface (see Fig. 1). To generate the raster pattern, the fast axis of the AFM nanopositioner is driven by a triangular signal and the slow axis is driven by a synchronized staircase or ramp signal. The triangular waveform contains all odd harmonics of the fundamental frequency. Although the amplitude of these harmonics is attenuated by a factor of $1 / n^{2}$, where $n$ is the number of the harmonic, a fast triangular

Manuscript received July 16, 2008; revised May 19, 2009; accepted August 18, 2009. Manuscript received in final form September 21, 2009. First published October 16, 2009; current version published August 25, 2010. Recommended by Associate Editor G. Guo. This work was supported in part by the Australian Research Council Center of Excellence for Complex Dynamic Systems and Control and by a University of Newcastle ECR grant.

Y. K. Yong and S. O. Reza Moheimani are with the School of Electrical Engineering and Computer Science, University of Newcastle, NSW 2308, Australia (e-mail: yuenkuan.yong@newcastle.edu.au; reza.moheimani@ newcastle. edu.au).

K. Liu is with Western Digital, Lake Forest, CA 92630 USA (e-mail: kexiu. liu@gmail.com).

Color versions of one or more of the figures in this brief are available online at http://ieeexplore.ieee.org.

Digital Object Identifier 10.1109/TCST.2009.2033201

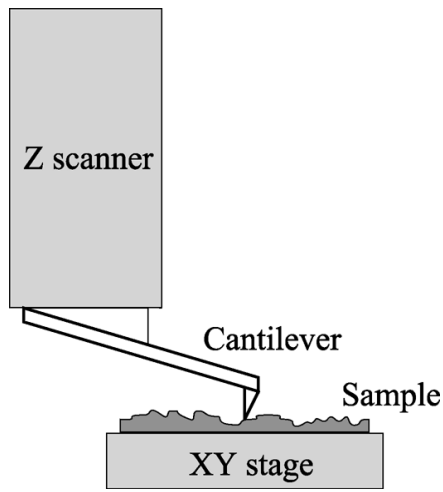

Fig. 1. Schematics of an AFM system documented in [18]. Unlike the conventional AFM, the $Z$ scanner is detached from the $X Y$ nanopositioner in this design.

may excite the resonance of the nanopositioner [8]. Due to the presence of the cross-coupling effect, the signal applied to the $X$-axis will corrugate the traced trajectory in the $X-Y$ plane.

The cross-coupling effect not only corrugates scans [9]-[11] but introduces artifacts to the image which can be mistaken as surface roughness [12]. Various calibration and correction techniques have been developed to reconstruct the distorted AFM images [10], [13], [14]. To avoid the post-processing of scans, a number of approaches have been implemented that minimize the $X Y$-to- $Z$ cross-coupling of AFM scanners. In [15], a kinematic model was derived to quantitatively analyze the scanning error of an AFM due to cross-coupling and to compensate for the error according to mathematical calculations. In [9], a model-based open loop controller is implemented to minimize the cross-coupling between the lateral and vertical axes of an AFM scanner. Also an iterative, inversion-based, feedforward control technique is proposed in [16] to reduce the $X$-to- $Z$ cross-coupling.

Apart from the cross-coupling between the $X Y$-plane and the $Z$-axis, AFM scanners also possess cross-couplings between their $X$ - and $Y$-axes, which can be observed from the experimental data presented in [17]. Atomic force microscopy is often performed at low scanning speeds. At such speeds the adverse effect of cross-coupling between the $X$ - and $Y$-axes is less significant. However, the cross-coupling effect becomes prominent during high-speed scans due to the excitation of mechanical resonances of the scanner. This cross-coupling is known to introduce measurement uncertainties in a scan [11].

A compliant $X Y$ nanopositioner with low cross-coupling effect is designed and presented in this brief. This flexure-based $X Y$ nanopositioner is aimed to be fitted into an AFM system similar to that proposed in [18]. In this AFM a sample is moved in the $X-Y$ plane using the planar $X Y$ nanopositioner while the AFM probe is maneuvered along the $Z$-axis by the $Z$ scanner (see Fig. 1). This design configuration practically removes the 
$X Y$-to- $Z$ cross-couplings. Furthermore, the dynamic performance of the $Z$ scanner is no longer limited by that of the $X Y$ nanopositioner.

To obtain undistorted scans at high-speeds, the $X$-to- $Y$ crosscoupling of the nanopositioner is minimized by 1) mechanically decoupling the two axes through the design of the structure and 2) implementing a $\mathrm{H}_{\infty}$ controller to reduce the cross-coupling especially during high-speed operations, and subsequently achieving accurate tracking performance.

The remainder of the brief is organized as follows. The mechanical design and finite-element-analysis (FEA) of the compliant nanopositioner are presented in Section II. In Section III, the experimental setup of the nanopositioning system is described. The system identification of the nanopositioner is presented in Section IV. Section V discusses the $\mathrm{H}_{\infty}$ controller design and the choice of necessary weighting functions. Experimental closed loop raster scan designs are presented in Section VI. Section VII presents the noise characterization of the closed loop system. Finally, Section VIII concludes this brief.

\section{Mechanical Design}

The design of the $X Y$ nanopositioner is based on the concept of compliant mechanisms where the requisite motions are generated through elastic deformations of flexible mechanical elements (flexures). Unlike conventional mechanisms, compliant mechanisms do not have gears, revolute and sliding joints. Consequently, the problems of wear, backlash, friction, and the need for lubrication are eliminated [19], [20]. This provides repeatable and smooth motions necessary for accurate nanoscale positioning. The design is monolithic (machined from a single piece of material) and has a parallel structural configuration. A parallel structure provides high mechanical stiffness, high motion accuracy, and high resonance frequencies, which makes the nanopositioner suitable for high-speed, accurate tracking applications [21]. Two piezoelectric stack actuators are incorporated into the nanopositioner, each providing the required input displacement to the $X$ - and $Y$-axes, respectively. They are used to drive the nanopositioner due to their capability of providing large forces, high accelerations and repeatable motions over a very large bandwidth [22]. The combination of the compliant mechanism design and the use of piezoelectric stack actuators allows for accurate nanoscale positioning with nanometer resolution at high scan speeds.

A key design specification for the nanopositioner is to minimize the effect of cross-coupling between the two axes of the device. As shown in Fig. 2 the nanopositioner consists of two main parts: 1) the inner section which consists of a nanopositioner and beam flexures and 2) the outer part which consists of amplification levers and circular flexures. The inner section is designed to minimize the cross-coupling between the $X$ - and $Y$ motions of the nanopositioner. At the inner section, the nanopositioner is held by four pairs of beam flexures. The flexures are arranged in such a way so that they are rigid along the direction of motion and are flexible in the axis perpendicular to the motion [23]. The FEA simulation of the $X$-to- $Y$ cross-coupling obtained using ANSYS is $-35 \mathrm{~dB}$, i.e., motions in the unactuated axis are about $1 / 56$ th of the actuated axis. The simulated

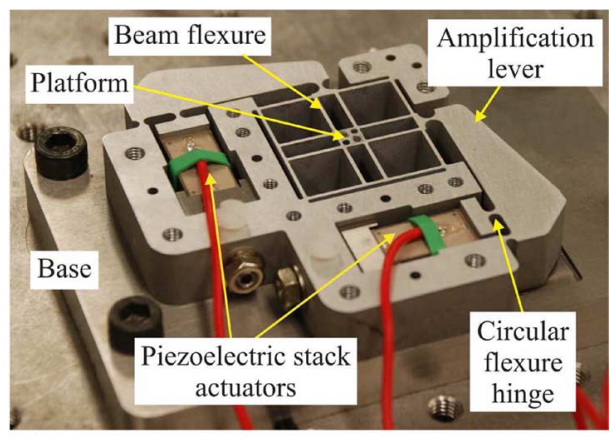

(a)

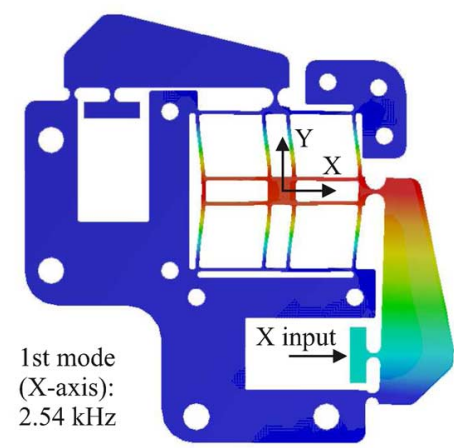

(b)

Fig. 2. (a) Flexure-based $X Y$ nanopositioner. The nanopositioner consists of the inner and outer sections. The inner section is designed to minimize the crosscoupling between the $X$ - and $Y$-axes. The outer section is designed to amplify the displacement of piezoelectric stack actuators. (b) FEA simulation of motions and resonance frequency of the nanopositioner along the $X$-axis. Simulations of the $Y$-axis are similar.

motion along the $X$-axis is shown in Fig. 2(b). At the outer section, an amplification lever is integrated into each axis of the nanopositioner to amplify the displacement of the piezoelectric stack actuator. The ANSYS estimation of the amplification ratio is about 2.5. The amplified motions are transferred to the inner section to displace the platform located at the inner section.

The nanopositioner was fabricated using the wire-electricaldischarge-machining (WEDM) technique due to its accuracy and precision [24]. Aluminum alloy 7075 with Young's modulus of $72 \mathrm{GPa}$ and a thickness of $12.8 \mathrm{~mm}$ was used to fabricate the nanopositioner. The chosen material thickness is large enough to ensure that the out-of-plane motion along the $Z$-axis is negligible. The final design of the nanopositioner is estimated to have its first resonance frequency at $2.5 \mathrm{kHz}$ and a cross-coupling of $-35 \mathrm{~dB}$ at both axes, which are deemed feasible for generating high-speed scans without encountering severe image distortions.

\section{EXPERIMENTAL SETUP}

The nanopositioner is mounted on a $10 \mathrm{~mm}$ steel plate that is rigidly held on an optical table. An aluminum block with a fine surface finish is mounted on the platform of the compliant nanopositioner and is used as a target for displacement sensing. It also serves as a stage for the placement of a sample. Two ADE Technologies 8810/2804 capacitive sensors, each with a static gain of $2.5 \mu \mathrm{m} / \mathrm{V}$, are placed adjacent to the aluminium block surfaces to measure the displacement along the $X$ - and $Y$-axes (see Fig. 3). The two Physik Instrumente piezoelectric 


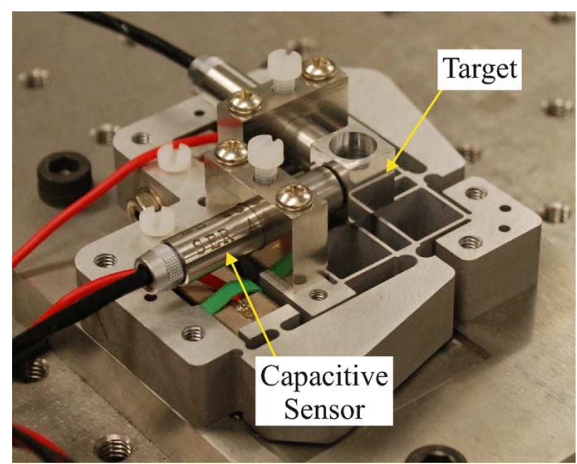

Fig. 3. Experimental nanopositioner setup. The $X$ and $Y$ displacements of the nanopositioner are measured using two capacitive sensors. The sensing target is made of aluminum and has a fine surface finish.

stack actuators (P-888.50) are used to generate motions along the two axes. These actuators operate over a voltage range of 0 to $100 \mathrm{~V}$ and produce a maximum stage motion of $25 \mu \mathrm{m}$ at each axis.

In this work, the nanopositioner is used to produce raster scans over a $10 \mu \mathrm{m} \times 10 \mu \mathrm{m}$ area. A triangular waveform and a synchronized staircase signal are generated to produce the raster scans. The triangular and the staircase signals drive the $X$ - and $Y$-axes piezoelectric stack actuators, respectively. Hysteresis is the main nonlinearity associated with piezoelectric materials. It is known to degrade the trace of the reference signal [25], [26]. Charge actuation has been implemented to substantially reduce the presence of hysteresis in piezoelectric actuators [27]-[29]. Since the $X$-axis piezoelectric actuator is driven, repetitively, over a relatively large range (i.e., $10 \mu \mathrm{m}$ ), positioning errors due to hysteresis effect at this axis are more significant than that of the $Y$-axis. A custom-built charge source capable of driving large capacitive loads is employed in this work to reduce the hysteresis effect in the $X$-axis. The charge amplifier has a gain of $126 \mu \mathrm{C} / \mathrm{V}$ and an equivalent voltage gain of 20 . The $Y$-axis piezoelectric stack actuator is driven to trace staircase signals. A voltage amplifier with a gain of -20 was used to drive the $Y$-axis actuator.

A dSPACE-1005 rapid prototyping system equipped with 16-bit ADC (DS2001) and DAC (DS2102) cards is used to implement a feedback controller in real time. A sampling frequency of $40 \mathrm{kHz}$ was chosen to ensure that there were no aliasing effects during the experiments.

\section{SYSTEM IDENTIFICATION}

The nanopositioner is a two-input two-output system. A block diagram is presented in Fig. 4 to clarify the associated gains in this system. The $X$-axis piezoelectric stack actuator was driven by a charge amplifier which has a gain of $126 \mu \mathrm{C} / \mathrm{V}$ and an equivalent voltage gain of 20 . A voltage amplifier with a gain of -20 was used to drive the $Y$-axis piezoelectric stack actuators.

To identify a linear model of the nanopositioner, within the bandwidth of interest, a HP 35670A dual channel spectrum analyzer was used to generate a band-limited random noise input $\left(u_{x}\right.$ and $\left.u_{y}\right)$ of amplitude $30 \mathrm{mVpk}$ within the frequency range of $10 \mathrm{~Hz}$ to $10 \mathrm{kHz}$. The signals were applied to the

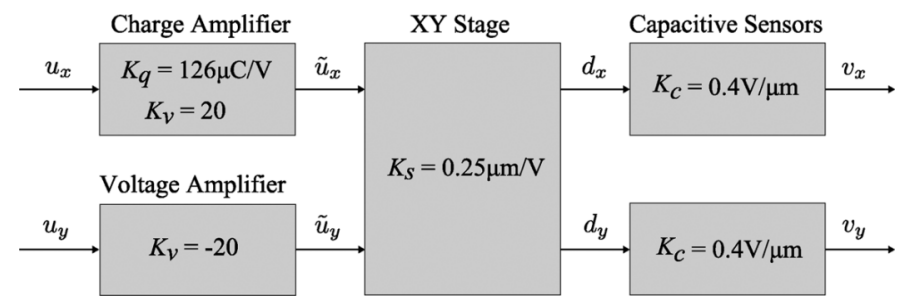

Fig. 4. Associated gains of the nanopositioning experimental setup. $u_{x}$ and $u_{y}$ are the reference inputs in volts generated by the spectrum analyzer. $\tilde{u}_{x}$ is the output of the charge amplifier in $\mu \mathrm{C}$ and $\tilde{u}_{y}$ is the output of the voltage amplifier in volts. $d_{x}$ and $d_{y}$ are the actual displacements of the nanopositioner in $\mu \mathrm{m}$, $v_{x}$ and $v_{y}$ are the capacitive sensor output in volts. The frequency responses are from input $u_{x}$ to output $v_{x}$ and from $u_{y}$ to $v_{y}$.

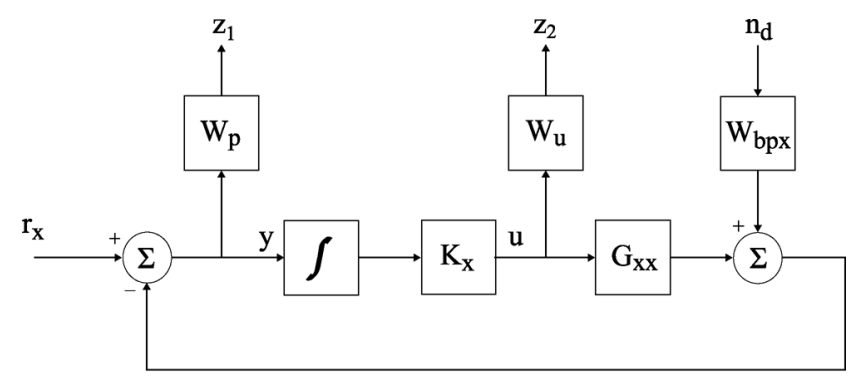

(a)

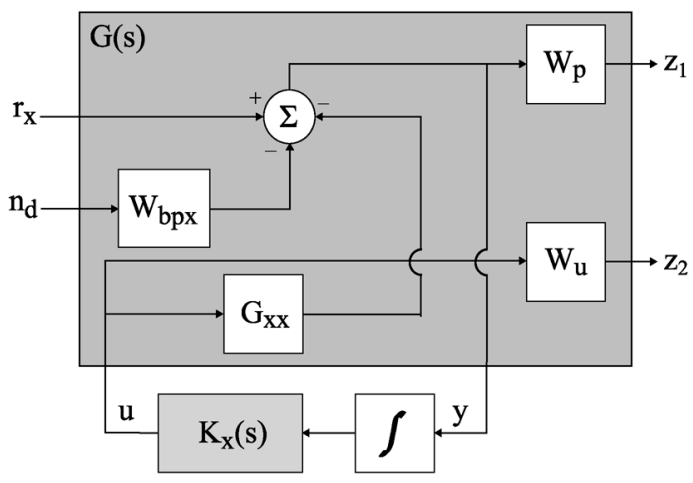

(b)

Fig. 5. Block diagram of the $X$-axis feedback loop. $Y$-axis feedback loop is quite similar. (a) Closed-loop system with weighting functions. (b) Equivalent block diagram of the closed-loop system which is cast into the $\mathrm{H}_{\infty}$ control design structure.

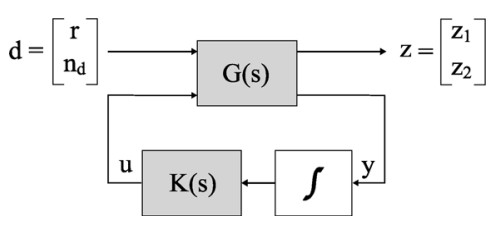

Fig. 6. Block diagram of the $\mathrm{H}_{\infty}$ controller.

$X$ - and $Y$-axes stack actuators using the charge and voltage amplifiers, respectively. The corresponding capacitive sensor measurements $\left(v_{x}\right.$ and $\left.v_{y}\right)$ were fed back to the spectrum analyzer to construct the frequency responses of the two channels. The frequency response functions of the system can be described as

$$
\left[\begin{array}{ll}
G_{x x}(j \omega) & G_{x y}(j \omega) \\
G_{y x}(j \omega) & G_{y y}(j \omega)
\end{array}\right]=\left[\begin{array}{ll}
\frac{v_{x}(j \omega)}{u_{x}(j \omega)} & \frac{v_{x}(j \omega)}{u_{y}(j \omega)} \\
\frac{v_{y}(j \omega)}{u_{x}(j \omega)} & \frac{v_{y}(j \omega)}{u_{y}(j \omega)}
\end{array}\right] .
$$



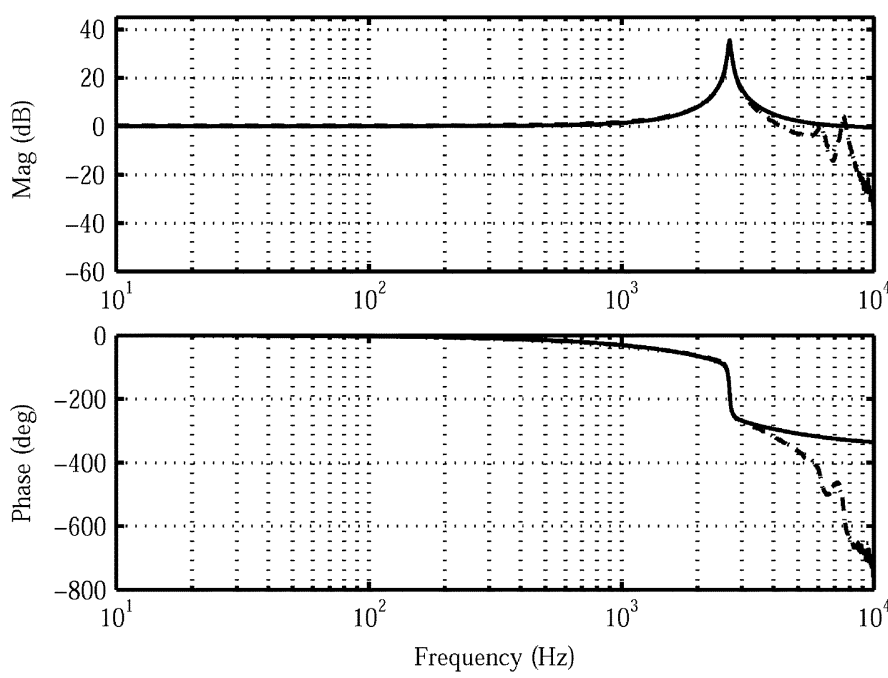

Fig. 7. Frequency responses of the measured $(--)$, the high order $(.$.$) and the$ second-order $(-)$ models of the nanopositioner ( $X$-axis). Frequency responses of the $Y$-axis is quite similar.

(a)

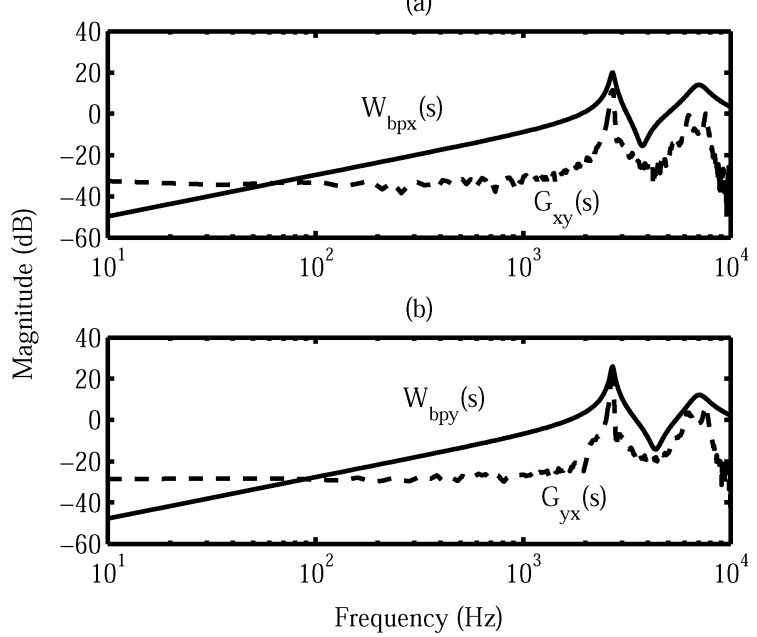

Fig. 8. Weighting functions incorporated into the $\mathrm{H}_{\infty}$ control design for crosscoupling attenuation. (a) $W_{b p x}(-)$ is tuned to the resonance frequencies of $G_{x y}(--)$. (b) $W_{b p y}(-)$ is tuned to the resonance frequencies of $G_{y x}(--)$.
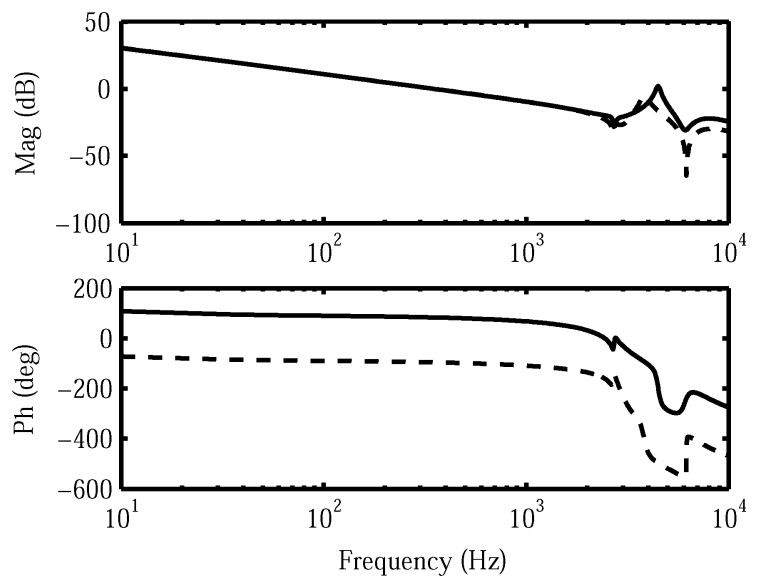

Fig. 9. Frequency responses of the $X(--)$ and $Y(-)$ axis controllers incorporating integral action.
Here, $u_{x}(j \omega), u_{y}(j \omega), v_{x}(j \omega)$, and $v_{y}(j \omega)$ denote the Fourier transforms of $u_{x}, u_{y}, v_{x}$, and $v_{y}$, respectively.

The measured open loop frequency responses are plotted in Fig. 10. The three resonance frequencies occur at 2.7, 6, and $7.6 \mathrm{kHz}$. The cross-coupling from $X$-to- $Y$ and $Y$-to- $X$ are -30 and $-35 \mathrm{~dB}$, respectively. The cross-coupling effect increased significantly near the three resonant peaks due to the excitation of mechanical resonance modes at each individual axis.

The open loop scanning performance of the nanopositioner is evaluated using raster signals. The nanopositioner is used to scan an area of $10 \mu \mathrm{m} \times 10 \mu \mathrm{m}$. The distance between the consecutive lines is $39 \mathrm{~nm}$, providing a total of 256 scan lines along the $Y$-axis. To evaluate the performance of the proposed high-speed raster scanning arrangement, triangular waves with fundamental frequencies of 40,60 , and $100 \mathrm{~Hz}$ are used to drive the $X$-axis actuator and the corresponding synchronized staircase signals are used to drive the $Y$-axis actuator. Fig. 11(a) shows the traces of the triangular and the synchronized staircase signals when the nanopositioner is operated in open loop. It can be observed that tracking performance of the nanopositioner when operated in this mode is unacceptably poor at high-speed.

To appreciate the problem, the desired and the actual trajectories traced by the nanopositioner in the $X-Y$ plane are plotted in Fig. 12(a). It can be observed that as the scan frequency speeds up, the positioning performance of the nanopositioner worsens. This is mainly due to the cross-coupling between $X$ and $Y$-axes. Although the nanopositioner is designed to have low cross-coupling effect, the higher order harmonics of the triangular signal excite the sharp resonances in the cross-coupling frequency response of the nanopositioner. Therefore, the signal applied to the $X$-axis corrugates the traced trajectory in the $X-Y$ plane.

Another effect that can be observed in Fig. 12(a) is the drift of the traced trajectory. This can be due to creep of the piezoelectric actuators and thermal drift of the nanopositioner. Piezoelectric stack actuators embedded into the nanopositioner are uni-polar actuators - to avoid depolarization, only positive voltages can be applied to them. These actuators are biased at $40 \mathrm{~V}$, and then $\mathrm{AC}$ signals are applied on top of the bias voltage. When operated in this mode, piezoelectric stack actuators are known to creep [30]. Thermal drift is also known to result in a similar drift in nanopositioning platforms [31]. To improve the tracking performance of the system and make it suitable for fast scans, these issues must be addressed. In the next section, we propose a control design framework that achieves this goal.

\section{V. $\mathrm{H}_{\infty}$ CONTROLler Design}

To perform fast and accurate raster scans, a nanopositioning system with high bandwidth, high resolution and good disturbance rejection capabilities is desirable. Previous section clearly illustrated that fast and accurate nanopositioning in open loop is not practical. To achieve accurate and fast nanopositioning, a $\mathrm{H}_{\infty}$ controller was designed and implemented on the system. The controller is designed to attenuate the cross-coupling effect between the two axes of the nanopositioner. It has high gain at low frequencies in order to substantially reduce 


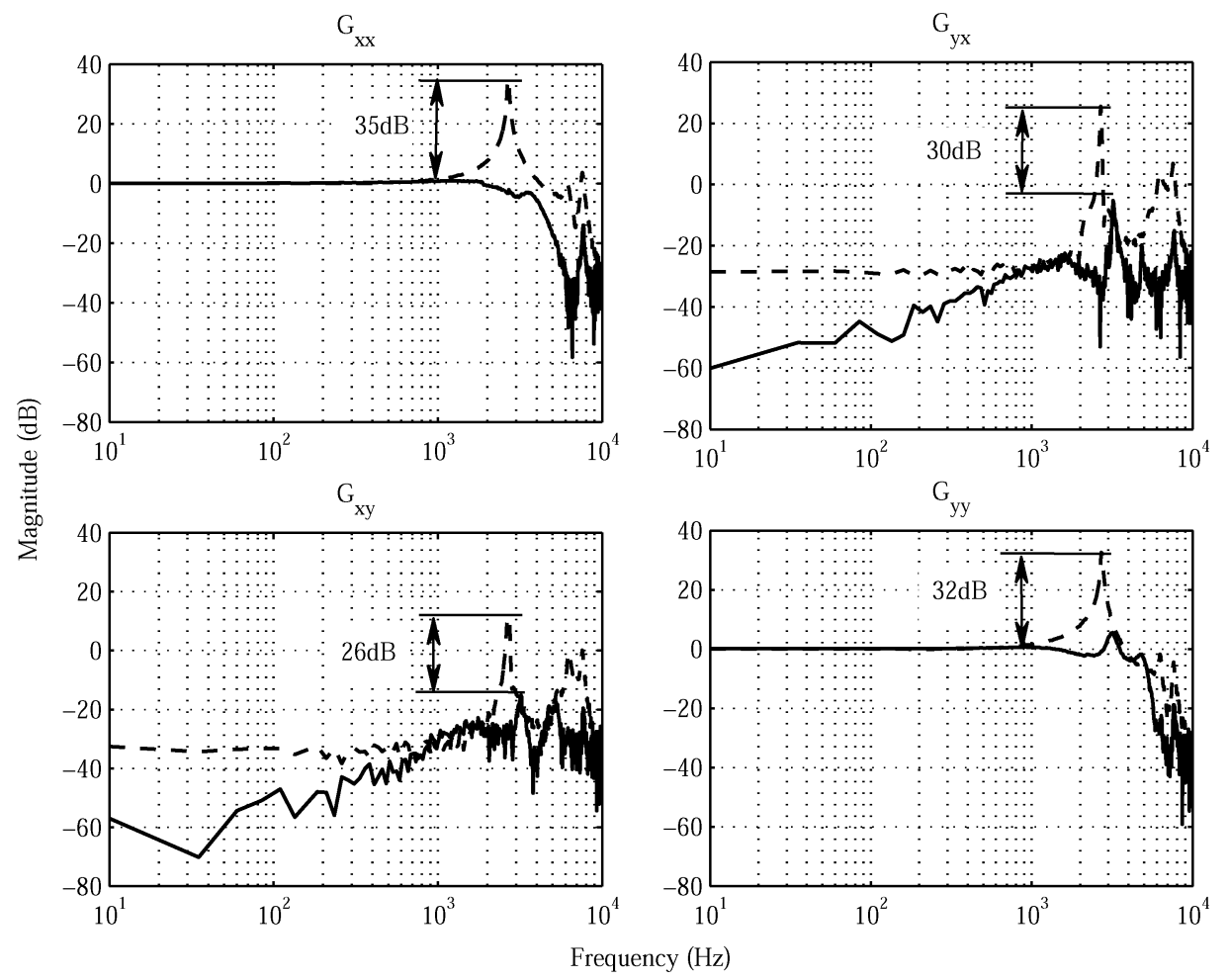

Fig. 10. Measured frequency responses showing magnitude (in decibels) of the open-loop (--) and closed-loop systems (-). In closed loop, the $X$-to- $Y$ and $Y$-to- $X$ cross-coupling are reduced substantially by 30 and $26 \mathrm{~dB}$, respectively, at the resonances of the nanopositioner. The controller imparts significant damping of the first resonant peaks by 35 and $32 \mathrm{~dB}$ at the $X$ - and $Y$-axis, respectively.

tracking errors. The controller was also designed to ensure the closed loop system has sufficient bandwidth to perform fast raster scans, i.e., to track fast triangular signals of up to $100 \mathrm{~Hz}$ in this case.

Fig. 5 shows the block diagram of the feedback controlled system with its associated weighting functions. Since the crosscoupling between the two axes is small at low frequencies (see Fig. 10), each positioning axis was considered as a SISO system. A controller was designed for each positioning axis and the cross-coupling effect was considered as an additional disturbance to each loop. The control design problem is cast into the standard $\mathrm{H}_{\infty}$ control design framework with the standard structure illustrated in Fig. 6. The input disturbance vector is defined as $d=\left[\begin{array}{ll}r & n_{d}\end{array}\right]^{T}$ and the control output vector is defined as $z=\left[\begin{array}{ll}z_{1} & z_{2}\end{array}\right]^{T}$, where $r$ is the reference signal, $n_{d}$ is the output disturbance that models the cross-coupling from the $Y$-axis, $u$ is the control input, $z_{1}$ and $z_{2}$ are the control outputs. The controller is designed to minimize $\left\|T_{z d}(s)\right\|_{\infty}$, the $\mathrm{H}_{\infty}$-norm of the transfer function from the input disturbance vector to the control output vector.

The 14th- and 12th-order plant models were initially identified for the $X$ - and $Y$-axes, respectively, using the frequency domain subspace-based modeling technique [32]. High order plant models lead to high order controllers that could be difficult to implement in real time. Consequently, second-order models $G_{x x}$ and $G_{y y}$ of the plant were used for controller design. These transfer functions are described in (2) and (3). Fig. 7 shows the frequency responses of the measured, the high order and the second order models of the nanopositioner for the $X$-axis, i.e., $G_{x x}$. The second-order models capture the dynamics of the system up to, and after the first resonance frequency with adequate accuracy

$$
\begin{aligned}
G_{x x}(s) & =\frac{1.563 s^{2}-3.623 \times 10^{4} s+4.851 \times 10^{8}}{s^{2}+340.4 s+2.84 \times 10^{8}} \\
G_{y y}(s) & =\frac{-1.492 s^{2}+3.459 \times 10^{4} s-4.632 \times 10^{8}}{s^{2}+344.3 s+2.905 \times 10^{8}} .
\end{aligned}
$$

As shown in Fig. 7, the reduced order models do not capture the second and the third resonant modes of the nanopositioner. For control design purposes the presence of these unmodeled modes is treated as output disturbances to the plant. These resonant modes are small in magnitude, compared with the first resonance of each axis, and appear at high frequencies, above 6 $\mathrm{kHz}$. To deal with these out of bandwidth modes the $\mathrm{H}_{\infty}$ controller must be designed to roll-off at high frequencies after the first resonant mode of each axis.

To minimize cross-coupling between the two axes, low order bandpass weighting functions $W_{b p_{x}}$ and $W_{b p_{y}}$ are introduced to $X$ - and $Y$-axis, respectively. Each weighting function is tuned to the resonance frequencies of its corresponding axis, as shown in Fig. 8. Due to the fact that the cross-coupling effect is more profound near the resonance frequencies, the weighting functions are designed to heavily suppress the cross-coupling at those frequencies. The magnitude of the weighting functions reduced at low frequencies in order to further minimize the 


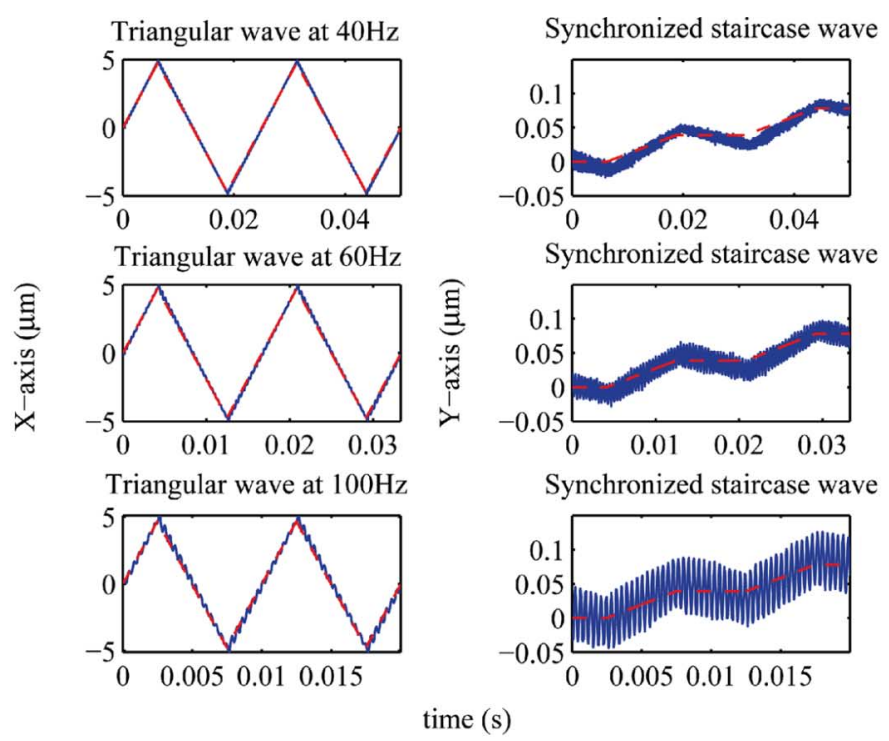

(a)

Triangular wave at $40 \mathrm{~Hz}$
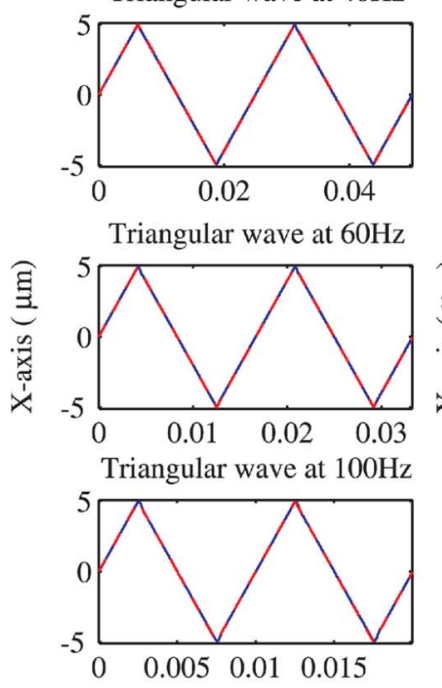

tinc(i)

(b)

Fig. 11. (a) Open- and (b) closed-loop tracking performance of triangular waves with fundamental frequencies of 40,60 , and $100 \mathrm{~Hz}$ and their corresponding synchronized staircase signals. Reference signals (--) and output signals (-) are plotted. In open loop, the resonant mode of the nanopositioner is excited by all three signals applied to the $X$-axis. The excitation effect can be observed at the $Y$-axis due to the cross-coupling effect. In closed loop, the effects of crosscoupling, piezoelectric creep and thermal drift are substantially reduced.

cross-coupling effect from $-35 \mathrm{~dB}(-35 \mathrm{~dB}$ was achieved from the mechanical design). The two weighting functions are

$$
\begin{aligned}
W_{b p x}= & \frac{52464.5973 s}{\left(s^{2}+848.2 s+2.878 \times 10^{8}\right)} \\
& \times \frac{\left(s^{2}+2133 s+5.54 \times 10^{8}\right)}{\left(s^{2}+8796 s+1.934 \times 10^{9}\right)} \\
W_{b p y}= & \frac{48757.518 s}{\left(s^{2}+678.6 s+2.878 \times 10^{8}\right)} \\
& \times \frac{\left(s^{2}+2938 s+7.461 \times 10^{8}\right)}{\left(s^{2}+8796 s+1.934 \times 10^{9}\right)} .
\end{aligned}
$$
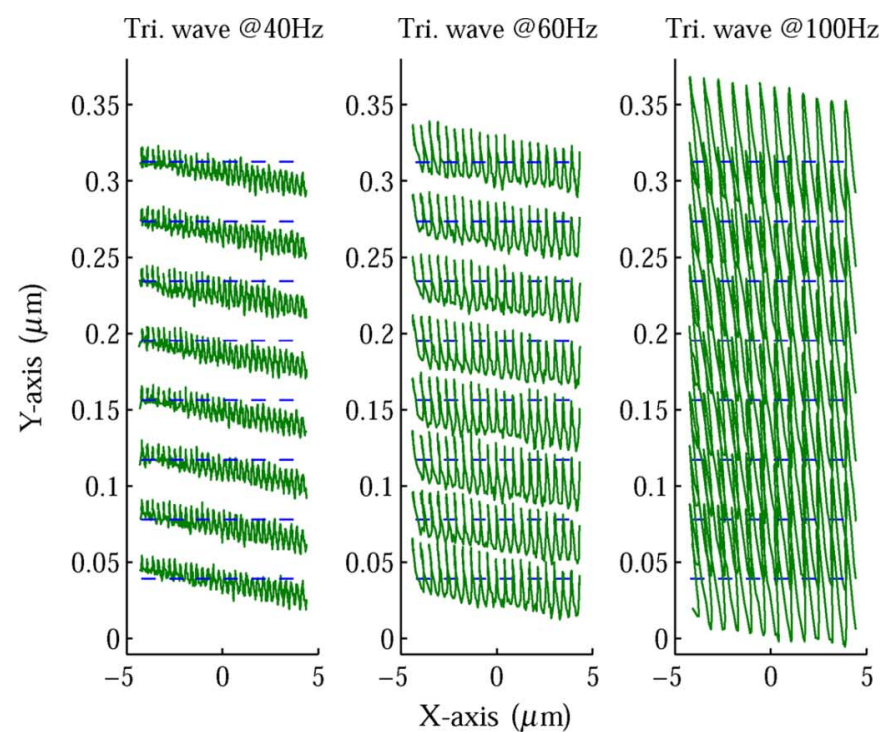

(a)

Tri.wave@40Hz Tri.wave@60Hz Tri.wave@100Hz

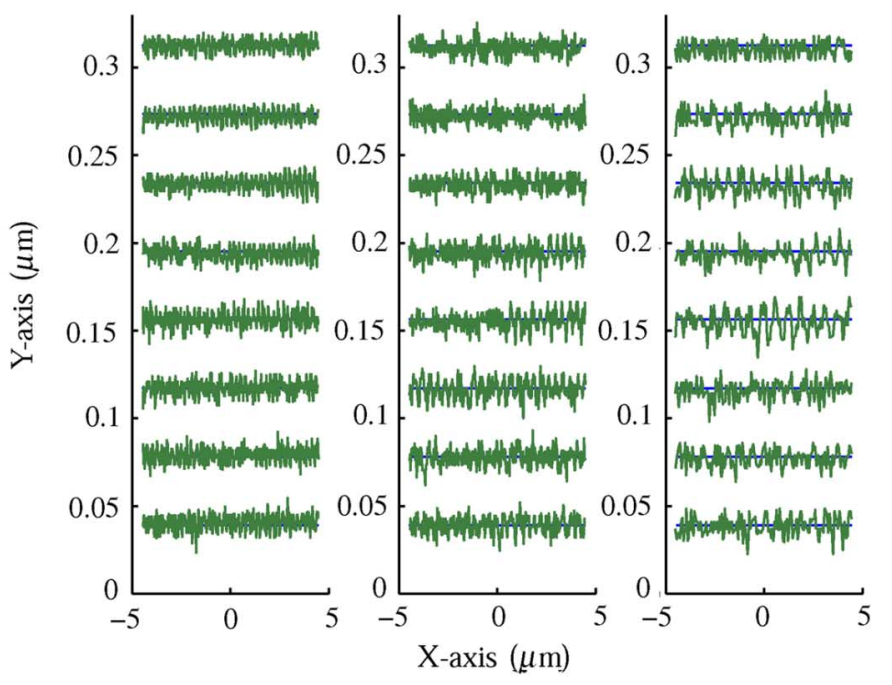

(b)

Fig. 12. (a) Open- and (b) closed-loop raster scan results. Reference signals $(--)$ and output signals $(-)$ are plotted. $90 \%$ of the scanning range which corresponds to $\pm 4.5 \mu \mathrm{m}$ along the $X$-axis is plotted. The scanning lines are 39 $\mathrm{nm}$ apart. In open loop, the inclination of scan lines is due to piezoelectric creep and thermal drift of the nanopositioner. The resonant mode of the nanopositioner is excited due to the triangular signal applied to the $X$-axis stack actuator and the cross-coupling causes the excitation effect to be seen at the $Y$-axis. In closed loop, the inclination of scan lines due to piezoelectric creep and thermal drift is eliminated. The cross-coupling effect is substantially reduced.

The weighting function $W_{u}$ is introduced to limit the level of control signal applied to each actuator. This is necessary to avoid actuator saturation during the operation of the device in closed loop. This weight was chosen as a constant and it tends to constrain the controller gain at high frequencies. The weighting function $W_{p}$ is introduced to enforce good tracking performance. Both $X$ - and $Y$-axes of the nanopositioner have very similar frequency responses. The cross-coupling between the two axes is also very similar. Thus, the same weight $W_{p}$ was used for both axes, i.e.,

$$
W_{p}=\frac{0.3162 s+6283}{s+19.87} .
$$


TABLE I

RMS ERRORS OF THE RASTER TRACKING PERFORMANCE IN OPEN AND Closed LoOP. ERrors Were CALCUlated By CONSIDERING 90\% OF THE SCANNING RANGE

\begin{tabular}{cccc}
\hline & \multicolumn{3}{c}{ Raster signal } \\
RMS error $(\mathrm{nm})$ & $40 \mathrm{~Hz}$ & $60 \mathrm{~Hz}$ & $100 \mathrm{~Hz}$ \\
\hline Open loop & 7.7 & 11.9 & 27.8 \\
Closed loop & 5.0 & 5.1 & 5.4 \\
\hline
\end{tabular}

An integrator was incorporated into the $\mathrm{H}_{\infty}$ controller to achieve satisfactory tracking of triangular and staircase signals. In Fig. 5, this integrator is shown cascaded to the plant. Fig. 9 displays frequency responses of the $X$ - and $Y$-axis controller with integral action.

Measured open and closed-loop frequency responses of the nanopositioner are shown in Fig. 10. The implemented controller attenuates the cross-coupling effect substantially, i.e., 30 and $25 \mathrm{~dB}$ attenuation for the $X$-to- $Y$ and $Y$-to- $X$ cross-coupling, respectively. The controller also achieved significant damping of the first resonant modes of the nanopositioner, i.e., by 35 and $32 \mathrm{~dB}$ at the $X$-and $Y$-axis, respectively.

\section{CLOSED-LOOP RASTER SCANS}

To evaluate the performance of the closed loop system in following raster patterns at high speeds, triangular waves with fundamental frequencies of 40,60 , and $100 \mathrm{~Hz}$ were used to drive the $X$-axis actuator and the corresponding synchronized staircase signals were used to drive the $Y$-axis actuator. Fig. 11(b) shows the desired and the actual traces of the $X$ - and $Y$-axis trajectories in closed loop. It can be observed that the damping introduced by the controller has a substantial effect on the quality of the scan. The resonant modes of the nanopositioner were significantly attenuated. Therefore, the harmonics of the triangular signals do not get amplified. Another important contributor to this high quality scan is the significant closed loop attenuation of cross-coupling between the two axes of the nanopositioner at low frequencies. Furthermore due to control action the effects of piezoelectric creep and thermal drift are substantially reduced.

Comparing the closed loop raster pattern in Fig. 12(b) with the open-loop raster pattern in Fig. 12(a), a significant improvement can be observed as a result of control action. To appreciate the improvement that has been achieved, the RMS errors corresponding to the open and closed loop scans for $90 \%$ of the scanning range (i.e., $9 \mu \mathrm{m}$ along the $X$-axis) are documented in Table I. It can be observed that the RMS error for all closed loop scans remain below $5.5 \mathrm{~nm}$. This is much smaller than the corresponding open-loop RMS tracking errors. In particular, it can be observed that by increasing the scan frequency the open loop RMS error increases substantially. However in closed loop the scan error appears to be dominated by stochastic noise, associated with capacitive sensors, that remains relatively unchanged at different scan frequencies. For the $100 \mathrm{~Hz}$ scan, the control strategy proposed here reduces the RMS error by $19 \%$ (from 27.8 to $5.4 \mathrm{~nm}$ ).

\section{NOISE CHARACTERIZATION AND ESTIMATION OF RESOLUTION}

One of the complications with operating nanopositioning systems in closed loop is the presence of sensor noise. Very often
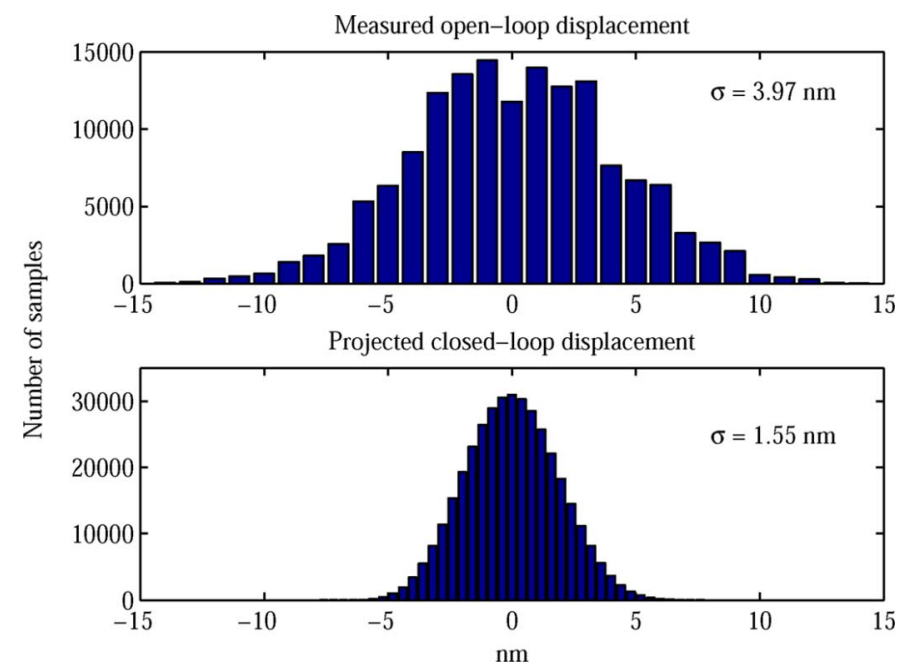

Fig. 13. Measured displacements of the open-loop system (top) and projected displacements of the closed-loop system (bottom). The standard deviation shows that the projected closed loop output is less noisy than the observed output.

a nanopositioner is required to operate with a precision that is comparable with the noise level experienced by its displacement sensor [33]. This is one of the main reasons why many practitioners prefer to operate nanopositioning platforms in open loop. However, as illustrated so far operating a nanopositioning device at high speeds and in open loop is not practical. Furthermore, although low frequency operation of a nanopositioner is possible, without a feedback controller issues such as piezoelectric creep and thermal drift can not be addressed in a satisfactory manner.

In the nanopositioning framework proposed here, the resolution of the closed-loop system is limited by the capacitive sensor noise. Motions of the nanopositioner that are below the sensor noise level cannot be detected, i.e., the "actual" output of the nanopositioner is not detectable at all times. In order to obtain a quantitative value of the "actual" output, the noise characterization techniques used in [34] and [33] were employed. The open loop sensor noise was measured when the nanopositioner was stationary. The noise data was then fed into a model of the closed loop system ${ }^{1}$ to obtain the projected displacement of the nanopositioner. Fig. 13 shows that standard deviation of the projected closed loop displacement is smaller than that of the measured open loop motion. This confirms that the "actual" output of the controlled nanopositioner is less noisy than the observed output. Based on this conclusion the scanning resolution of the platform can be improved by, at least, a factor of 2 , allowing for a total of 512 lines scan. This amounts to $19.5 \mathrm{~nm}$ distance between every two consecutive scan lines along the $Y$-axis.

\section{CONCLUSION}

The main goal of this brief was to build a fast nanoscale positioning platform with somewhat decoupled motion axes for high speed atomic force microscopy. This was done by: 1) designing a $X Y$ flexure-based nanopositioning device with low

\footnotetext{
${ }^{1}$ The closed-loop model is identified using the subspace-based modeling tech-
} nique from the measured closed-loop FRF. 
cross-coupling between its two axes and 2) designing and implementing a controller to minimize the positioning error arising from cross-couplings and to damp the first mechanical resonant peak. It was shown that fast operation of the device in open loop is not possible. The main complicating factor was identified to be the cross-coupling that exists between the two axes of the nanopositioner. Other contributors to positioning error were found to be piezoelectric creep and thermal drift. $\mathrm{A} \mathrm{H}_{\infty}$ controller was designed to minimize the effect of resonant modes of the nanopositioner on tracking high speed raster signals. This was achieved by adding substantial damping to lightly damped poles of the system. Furthermore, real time implementation of the controller resulted in the removal of the adverse effects of thermal drift and piezoelectric creep on the generated raster patterns. Experimental raster scans over an area of $10 \mu \mathrm{m} \times 10$ $\mu \mathrm{m}$, with a resolution of $39 \mathrm{~nm}$, at 40,60 , and $100 \mathrm{~Hz}$ scan frequencies demonstrated a significant improvement due to the presence of feedback controller. The achieved resolution allows for generation of a $256 \times 256$ AFM image. A noise analysis of the platform suggested that the resolution can be substantially increased, allowing for an image with a resolution of, at least, $512 \times 512$ pixels over the same area.

\section{ACKNOWLEDGMENT}

The research was performed at the Laboratory for Dynamics and Control of Nanosystems at the University of Newcastle.

\section{REFERENCES}

[1] G. Binnig and H. Rohrer, "The scanning tunneling microscope," Scientific American, vol. 253, pp. 50-56, 1986.

[2] S. Gonda, T. Kurosawa, and Y. Tanimura, "Mechanical performances of a symmetrical, monolithic three-dimensional fine-motion stage for nanometrology," Meas. Sci. Technol., vol. 10, pp. 986-993, 1999.

[3] T. Ando, N. Kodera, T. Uchihashi, A. Miyagi, R. Nakakita, H. Yamashita, and K. Matada, "High-speed atomic force microscope for capturing dynamic behavior of protein molecules at work," $e$-Journal of Surface Science and Nanotechnology, vol. 3, pp. 384-392, 2005.

[4] P. Vettiger, G. Cross, M. Despont, U. Drechsler, U. Dürig, B. Gotsmann, W. Häberle, M. A. Lantz, H. E. Rothuizen, R. Stutz, and G. K. Binnig, "The "milipede"-Nanotechnology entering data storage," IEEE Trans. Nanotechnology, vol. 1, no. 1, pp. 39-55, 2002.

[5] B. Bhushan, Handbook of Micro/Nanotribology, 2nd ed. Boca Raton, USA: CRC, 1999

[6] A. Sebastian, A. Pantazi, S. O. R. Moheimani, H. Pozidis, and E. Eleftheriou, "Achieving sub-nanometer precision in a MEMS storage device during self-servo write process," IEEE Transactions on Nanotechnology, vol. 7, no. 5, pp. 586-595, 2008.

[7] J. J. Gorman, N. G. Dagalakis, and B. G. Boone, "Multi-loop control of nanopositioning mechanism for ultra-precision beam steering," in Proc. SPIE Conf. Free-Space Laser Communication and Active Laser Illumination III, San Diego, CA, 2003, vol. 5160, pp. 170-181.

[8] S. O. R. Moheimani, "Invited review article: Accurate and fast nanopositioning with piezoelectric tube scanners: Emerging trends and future challenges," Review of Scientific Instruments, vol. 79, no. 7, p. 071101, July 2008, article Number: 071101.

[9] G. Schitter and A. Stemmer, "Model-based signal conditioning for high-speed atomic force and friction force microscopy," Microelectronic Engineering, vol. 67-68, pp. 938-944, 2003.

[10] Y. Sun and J. H. L. Pang, "AFM image reconstruction for deformation measurements by digital image correlation," Nanotechnology, vol. 17, pp. 933-939, 2006.

[11] D. Ricci and P. C. Braga, "Recognizing and avoiding artifacts in AFM imaging," in Atomic Force Microscopy: Biomedical Methods and Applications, ser. Methods in Molecular Biology. : Humana Press, 2004, vol. 242, pp. 25-37.
[12] O. M. El Rifai and K. Youcef-Toumi, "Coupling in piezoelectric tube scanners used in scanning probe microscopes," in Proceedings of the American Control Conference, Arlington, VA, June 25-27, 2001.

[13] J. Akila and S. S. Wadhwa, "Correction for nonlinear behavior of piezoelectric tube scanners used in scanning tunneling and atomic force microscopy," Rev. Sci. Instrum., vol. 66, no. 3, pp. 2517-2519, 1995.

[14] J. Garnaes, L. Nielsen, K. Dirscherl, J. F. Jorgensen, J. B. Rasmussen, P. E. Lindelof, and C. B. Sorensen, "Two-dimensional nanometer-scale calibration based on one-dimensional gratings," Applied Physics A, vol. 66, pp. S831-S835, 1998.

[15] X. Tian, N. Xi, Z. Dong, and Y. Wang, "System errors quantitative analysis of sample-scanning afm," Ultramicroscopy, vol. 105, pp. 336-342, 2005.

[16] S. Tien, Q. Zou, and S. Devasia, "Iterative control of dynamics-coupling-caused errors in piezoscanners during high-speed AFM operation," IEEE Trans. Contr. Syst. Tech., vol. 13, no. 6, pp. 921-931, 2005.

[17] B. Bhikkaji, M. Ratnam, and S. O. R. Moheimani, "PVPF control of piezoelectric tube scanners," Sensors and Actuators A, vol. 135, pp. 700-712, 2007.

[18] J. Kwon, J. Hong, Y.-S. Kim, D.-Y. Lee, K. Lee, S.-M. Lee, and S.-I. Park, "Atomic force microscope with improved scan accuracy, scan speed, and optical vision," Rev. Sci. Instrum., vol. 74, no. 10, pp. 4378-4383, 2003.

[19] L. Howell, Compliant Mechanisms. : John Wiley \& Sons, Inc., 2001.

[20] Y. K. Yong, T.-F. Lu, and D. C. Handley, "Review of circular flexure hinge design equations and derivation of empirical formulations," Precision Engineering, vol. 32, no. 2, pp. 63-70, 2008.

[21] Y. K. Yong and T.-F. Lu, "The effect of the accuracies of flexure hinge equations on the output compliances of planar micro-motion stages," Mechanism and Machine Theory, vol. 43, pp. 347-363, 2008.

[22] S. Salapaka, A. Sebastian, J. P. Cleveland, and M. V. Salapaka, "High bandwidth nano-positioner: A robust control approach," Rev. Sci. Instrum., vol. 73, no. 9, pp. 3232-3241, 2002.

[23] J. H. Kindt, G. E. Fantner, J. A. Cutroni, and P. K. Hansma, "Rigid design of fast scanning probe microscopes using finite element analysis," Ultramicroscopy, vol. 100, pp. 259-265, 2004.

[24] K. H. Ho, S. T. Newman, R. S. , and R. D. Allen, "State of the art in wire electrical discharge machining (WEDM)," International Journal of Machine Tools and Manufacture, vol. 44, no. 12-13, pp. 1247-1259, 2004.

[25] B. Mokaberi and A. A. G. Requicha, "Compensation of scanner creep and hysteresis for AFM nanomanipulation," IEEE Trans. on Automation Science and Engineering, vol. 5, no. 2, pp. 197-206, Apr. 2008.

[26] D. Croft, G. Shedd, and S. Devasia, "Creep, hysteresis, and vibration compensation for piezoactuators: Atomic force microscopy application," Journal of Dynamic Systems, Measurement, and Control, vol. 123, no. 1, pp. 35-43, 2001.

[27] A. J. Fleming and S. O. R. Moheimani, "A grounded-load charge amplifier for reducing hysteresis in piezoelectric tube scanners," Rev. Sci. Instrum., vol. 76, no. 7, p. 073707, 2005.

[28] A. J. Fleming and S. O. R. Moheimani, "Sensorless vibration suppression and scan compensation for piezoelectric tube nanopositioners," IEEE Trans. Contr. Syst. Tech., vol. 14, no. 1, pp. 33-44, 2006.

[29] A. J. Fleming and S. O. R. Moheimani, "Precision current and charge amplifiers for driving highly capacitive piezoelectric loads," Electronics Letters, vol. 39, no. 3, pp. 282-284, Feb. 2003.

[30] H. M. S. Georgiou and R. B. Mrad, "Dynamic electromechanical drift model for PZT," Mechatronics, vol. 18, pp. 81-89, 2008.

[31] B. Mokaberi and A. A. G. Requicha, "Drift compensation for automatic nanomanipulation with scanning probe microscopes," IEEE Trans. on Automation Science and Engineering, vol. 3, no. 9, pp. 199-207, 2006.

[32] T. McKelvey, H. Akcay, and L. Ljung, "Subspace based multivariable system identification from frequency response data," IEEE Trans. on Automatic Control, vol. 41, no. 7, pp. 960-978, July 1996.

[33] S. S. Aphale, B. Bhikkaji, and S. O. R. Moheimani, "Minimizing scanning errors in piezoelectric stack-actuated nanopositioning platforms," IEEE Trans. Nanotechnology, vol. 7, no. 1, pp. 79-90, 2008.

[34] A. Sebastian and S. M. Salapaka, "Design methodologies for robust nano-positioning," IEEE Trans. Contr. Syst. Tech., vol. 13, pp. 868-876, 2005. 